\title{
RESOLUÇÃO ANALÍTICA DA EQUAÇÃO DE DIFUSÃO-ADVECÇÃO CONSIDERANDO O TERMO DE CONTRAGRADIENTE APLICADO À DISPERSÃO DE POLUENTES NA ATMOSFERA
}

\author{
Cristiane Venzke ${ }^{1}$, Camila P. Costa ${ }^{2}$, Rejane Pergher ${ }^{2}$ \\ ${ }^{1}$ Mestranda do Programa de Modelagem Matemática - UFPEL \\ ${ }^{2} \mathrm{UFPEL}$ - IFM - DME \\ crisvenzke@hotmail.com
}

\section{RESUMO}

Neste trabalho iremos empregar uma nova formulação para o termo de contragradiente, proposta por Roberti et al. (2004), ao resolver a equação de difusão-advecção na Camada Limite Convectiva (CLC) através do método ADMM (Advection Diffusion Multilayer Method). Consideramos uma modelagem mais realística, levando em conta os efeitos do termo de contragradiente caracterizado pelo transporte não-local da dispersão.

\section{ABSTRACT}

In this work, we apply a new formulation for the countergradient term proposed by Roberti et al. (2004), to solve the advection-diffusion equation in the Convective Boundary Layer (CBL) by the ADMM method (Advection Diffusion Multilayer Method). In this way, we consider a more realistic model, taking into account the effects of the countergradient term characterized by nonlocal transport of the dispersion.

\section{INTRODUÇÃO}

Uma forma de estimar a concentração de poluentes considerando, de um modo mais realístico, a estrutura complexa da turbulência na Camada Limite Convectiva (CLC) é levando em conta o caráter não-local da dispersão, utilizando o termo de contragradiente (Arya, 1999).

Sabemos que há diversas parametrizações sendo utilizadas para o termo de contragradiente. Roberti et al. (2004) desenvolveram uma nova formulação onde a representação do contragradiente é deduzida através de um problema inverso.

No presente trabalho, empregamos esta parametrização ao resolver a equação de difusão-advecção na CLC através do método conhecido como Advection Diffusion Multilayer Method (ADMM - Moreira et al., 2006) com o objetivo de, além de ampliar a aplicabilidade do método, verificar se o termo de contragradiente deduzido por Roberti et al. (2004), melhora a modelagem.

\section{METODOLOGIA}

A modelagem da dispersão de poluentes na atmosfera pode ser dada pela equação bidimensional de difusão-advecção:

$$
u \frac{\partial \bar{c}}{\partial x}=-\frac{\partial \overline{w^{\prime} c^{\prime}}}{\partial z}+S
$$


onde $\bar{c}$ representa a concentração média integrada lateralmente, $u$, a velocidade média do vento na direção $x, \overline{w^{\prime} c^{\prime}}$, o fluxo turbulento de poluentes na direção $z$ e $S$, o termo fonte, sujeita às condições de contorno de fluxo nulo no solo e no topo da CLC.

Para resolver o problema de fechamento da Eq. (1) utilizamos a relação proposta por Deardorff (1966)

$$
\overline{w^{\prime} c^{\prime}}=-K_{z}\left(\frac{\partial \bar{c}}{\partial z}-\gamma\right),
$$

onde $K_{z}$ é o coeficiente de difusão turbulento e ${ }^{\gamma}$, o termo de contragradiente.

Substituindo a Eq. (2) na Eq. (1) e, considerando que o termo fonte será adiante inserido em uma das condições do problema, obtemos:

$$
u \frac{\partial \bar{c}}{\partial x}=-\frac{\partial}{\partial z}\left[-K_{z}\left(\frac{\partial \bar{c}}{\partial z}-\gamma_{c}\right)\right]
$$

Muitas parametrizações para o termo de contragradiente são utilizadas e encontradas na literatura. Neste trabalho, utilizamos a parametrização proposta por Roberti et al. (2004):

$$
\gamma_{c}=0,085 \cdot\left(\frac{q_{w}}{\Psi}\right)\left(\frac{h}{z}\right)^{2 / 3} \cdot \frac{1}{h} \cdot \bar{c}
$$

onde $\quad q_{w}=z /\left[0.594 h\left(1-e^{[-4(z / h)-0.00038(z / h)]}\right)\right] \quad$ é a função de estabilidade, $\Psi=0.913$ é função de dissipação adimensional e $h$ é a altura da CLC. Para resolver a Eq. (3), propomos denotar: $\quad \alpha=0,085 \cdot\left(\frac{q_{w}}{\Psi}\right)\left(\frac{h}{z}\right)^{2 / 3} \cdot \frac{1}{h} \quad$ e, $\quad$ então,
temos $\cdot \gamma_{c}=\alpha(z) \cdot \bar{c}(x, z)$

Logo, para encontrar a concentração ${ }^{\bar{c}}$, considerando o termo de contragradiente, devemos resolver o problema:

$$
\begin{aligned}
& u \frac{\partial \bar{c}}{\partial x}=-\frac{\partial}{\partial z}\left[-K_{z}\left(\frac{\partial \bar{c}}{\partial z}-\alpha \cdot \bar{c}\right)\right] \\
& K_{z}\left(\frac{\partial \bar{c}}{\partial z}-\alpha \cdot \bar{c}\right)=0 \text { em } z=0, h \\
& u \bar{c}=Q \cdot \delta\left(z-H_{s}\right) \text { em } x=0
\end{aligned}
$$

onde $Q$ é a taxa de emissão da fonte na altura $H_{s}$ e $\delta$ é a função delta de Dirac.

A solução será obtida através do ADMM, amplamente empregado na resolução da equação de difusão-advecção.

Para tanto, discretizamos a CLC em N subcamadas e tomamos os valores médios para os parâmetros $K_{z}, u$ e $\alpha$ dependentes da altura $z$ em cada subcamada $\Delta z_{n}=\left[z_{n-1}, z_{n}\right]$ :

$$
K_{z_{n}}=\frac{1}{\Delta z_{n}} \int_{z_{n-1}}^{z_{n}} K_{z}(z) d z \quad u_{n}=\frac{1}{\Delta z_{n}} \int_{z_{n-1}}^{z_{n}} u(z) d z \text { e } \alpha_{n}=\frac{1}{\Delta z_{n}} \int_{z_{n-1}}^{z_{n}} \alpha(z) d z
$$


Também, supomos contato perfeito entre as $\mathrm{N}$ subcamadas, com as seguintes condições de continuidade para a concentração e fluxo na interface em $z=z_{n}$ e $n=1,2, \ldots,(N-1)$ :

$$
\bar{c}_{n}=\bar{c}_{n+1}, \mathrm{e} \quad K_{z_{n}}\left(\frac{\partial \bar{c}_{n}}{\partial z}-\alpha_{n} \cdot \bar{c}_{n}\right)=K_{z_{n+1}}\left(\frac{\partial \bar{c}_{n+1}}{\partial z}-\alpha_{n+1} \cdot \bar{c}_{n+1}\right) .
$$

Portanto, a fim de obter a solução do problema (5), devemos resolver $\mathrm{N}$ problemas da forma:

$$
\begin{gathered}
u_{n} \frac{\partial \bar{c}_{n}}{\partial x}=K_{z_{n}} \frac{\partial^{2} \bar{c}_{n}}{\partial z^{2}}-K_{z_{n}} \cdot \alpha_{n} \frac{\partial \bar{c}_{n}}{\partial z} \\
K_{z_{n}}\left(\frac{\partial \bar{c}_{n}}{\partial z}-\alpha_{n} \cdot \bar{c}_{n}\right)=0 \text { em } z=0, h \\
u_{n} \bar{c}_{n}=Q \cdot \delta\left(z-H_{s}\right) \text { em } x=0
\end{gathered}
$$

O sistema (8) é solucionado analiticamente, através da transformada de Laplace em $x$, que resulta numa equação diferencial ordinária que depende apenas de $z$, cuja solução é

$$
\begin{aligned}
\overline{C_{n}}(s, z)=A_{n} \cdot e^{\left[\alpha_{n}+R_{n}\right] / 2}+B_{n} \cdot e^{\left[\alpha_{n}-R_{n}\right] / 2}+ \\
+\frac{Q}{R_{n} K_{z_{n}}}\left(e^{\left[\alpha_{n}-R_{n} \frac{\left(z-H_{s}\right)}{2}\right.}-e^{\left[\alpha_{n}+R_{n}\right] \frac{\left(z-H_{s}\right)}{2}}\right) H\left(z-H_{s}\right)
\end{aligned}
$$

Onde $R_{n}=\sqrt{\alpha_{n}{ }^{2}+\frac{4 u_{n} \cdot s}{K_{z_{n}}}}, \quad \alpha_{n}=\frac{1}{\Delta z_{n}} \int_{z_{n-1}}^{z_{n}} \alpha(z) d z, \alpha(z)=\frac{0,085}{h^{1 / 3} z^{2 / 3}}\left(\frac{q_{w_{n}}}{\Psi}\right)$ e $\quad H \quad$ a função de
Heaviside.

Posteriormente, aplicamos as condições de contorno e de interface, para definir as constantes de integração $A_{n} \mathrm{e}^{B_{n}}$.

Encontramos a concentração de poluentes aplicando a transformada inversa de Laplace, gerando uma solução integral:

que será resolvida numericamente.

$$
\begin{aligned}
\bar{c}_{n}(x, z)= & \frac{1}{2 \pi i} \int_{\xi-i \infty}^{\xi+i \infty} e^{s x}\left\{A_{n} \cdot e^{\left[\alpha_{n}+R_{n}\right] / 2}+B_{n} \cdot e^{\left.\left[\alpha_{n}-R_{n}\right]\right] / 2}+\right. \\
& \left.+\frac{Q}{R_{n} K_{z_{n}}}\left(e^{\left[\alpha_{n}-R_{n}\right]} \frac{\left(z-H_{s}\right)}{2}-e^{\left[\alpha_{n}+R_{n}\right]} \frac{\left(z-H_{s}\right)}{2}\right) \cdot H\left(z-H_{s}\right)\right\} d s,
\end{aligned}
$$




\section{CONSIDERAÇÕES FINAIS}

Podemos perceber que essa nova formulação do termo de contragradiente proposta por Roberti et al., (2004), não gerou grandes dificuldades na aplicação do método ADMM para resolver a equação de difusão-advecção que modela a dispersão de poluentes. Essa nova abordagem considera uma modelagem mais realística levando em conta os efeitos do termo de contragradiente caracterizado pelo transporte não-local da dispersão. O próximo passo será avaliar a solução obtida comparando com resultados disponíveis na literatura e fazer uma análise crítica sobre a física que embasa essa abordagem.

AGRADECIMENTOS: Agradecemos à CAPES pelo suporte financeiro.

\section{REFERÊNCIAS}

Arya, S.P., 1999. "Air Pollution Meteorology and Dispersion”, Oxford University Press, 310p.

Costa, C.P., Vilhena, M.T., Moreira, D.M., Tirabassi, T. 2006. "Semi-analytical solution of the steady three-dimensional advection-diffusion equation in the planetary boundary layer", Atmos. Environ., 40, 5659-5669.

Deardorff, J.W., 1966. "The countergradient heat flux in the lower atmosphere and in the laboratory", J. Atmos. Sci., 23, 503-506.

Moreira, D.M., Vilhena, M.T., Tirabassi, T., Costa, C.P., and Bodmann, B., 2006. "Simulation of Pollutant Dispersion in the Atmosphere by the Laplace Transform: The ADMM Approach", Water, Air, \& Soil Pollution, 177, 411-439.

$\mathrm{R}$

oberti, D.R., Campos Velho, H.F., Degrazia, G.A., 2004. "Identifing Counter-gradient Term in Atmospheric Convective Boundary Layer", Inverse Problems in Science and Engineering, 12(3), 329-339. 